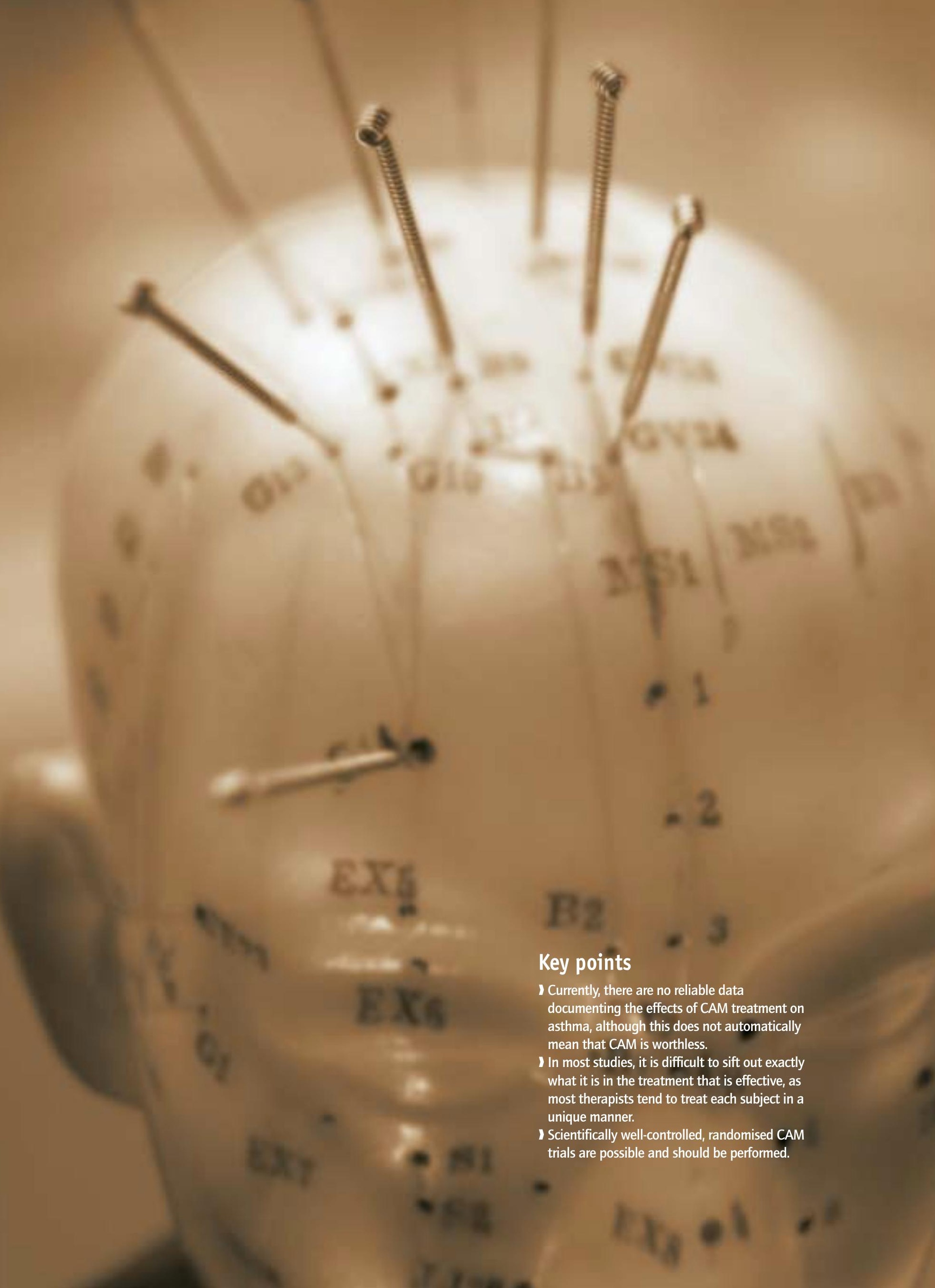




\section{Complementary and alternative treatment of asthma}

\section{Educational aims}

I To review the range of complementary and alternative (CAM) treatments currently available.

I To provide information about the systematic overviews in the Cochrane database concerning CAM and asthma.

\section{Summary}

Asthma and allergies are chronic conditions that cause considerable distress. In most cases, the use of modern medical therapy can control these conditions; however, there is still no cure for asthma. Therefore, many patients and, in the case of children, many parents seek CAM. This is often, but not always, concealed from the doctor treating the patient. Traditionally, there has been an antagonistic relationship between CAM and orthodox medicine, and this is why many patients do not inform their doctors that they are seeking CAM. This study aims to assess the current clinical medical value of CAM.

In orthodox medicine, there is an estab-

lished tradition of demonstrating whether a drug is effective or not by means of randomised, placebo-controlled trials. The placebo effect has been well established and, in most contexts, is estimated as accounting for as much as $30 \%$ of the total clinical effect. The attitude of the therapist treating the patient has, of course, considerable significance, as a charismatic therapist providing a positive treatment environment can additionally reinforce the placebo effect. On its own, this is not the field of the alternative therapist, but is commonly called "the bedside manner", whether practised consciously or not.

Over the last 10years, evidence-based medicine has become fashionable. Previously, guidelines were based on the knowledge of experts and so-called "opinion leaders". However, guidelines for treatment are now being developed from existing knowledge, which is based on research. Existing research is ranked according to an established/recognised system, and guidelines on how to write guidelines have been created [1]. Systematic reviews are constantly being developed and, through international cooperation, systematic reviews of special or particular subjects or substances are collected in databases. The Cochrane database contains such systematic overviews, in addition to overviews of randomised clinical trials. In the Cochrane collaboration, physicians are grouped according to subject, e.g. within respiratory diseases.
L. Bjermer

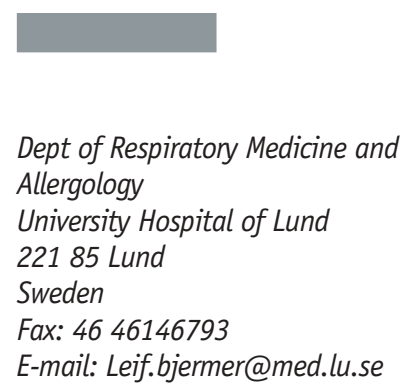


These kinds of systematic overviews have also been carried out with regards to complementary or alternative medicine (CAM), and the present review represents an attempt to provide information about the systematic overviews available concerning CAM and asthma in the Cochrane database. In total, 10 systematic reviews were found in the Cochrane database that relate to CAM. Most of these reviews are concerned with asthma; however, there will also be some reference to one systematic review concerning the use of acupuncture in smoking cessation, as this has particular relevance for respiratory diseases.

The Cochrane database can be accessed via the Internet at the following web address: http://www.cochrane.org/reviews/clibintro.htm

Table 1 shows the systematic reviews found in the Cochrane database for asthma.

\section{Table 1}

\section{Systematic reviews on the effects of CAM for asthma within the Cochrane database}

\begin{tabular}{|lll}
\hline Title & First author & Ref. \\
Homeopathy for chronic asthma & MCCARNEY & {$[2]$} \\
Manual therapy for asthma & HONDRAS & {$[4]$} \\
Acupuncture for chronic asthma & MCCARNEY & {$[5]$} \\
Ionisers for chronic asthma & BLACKHALL & {$[6]$} \\
Speleotherapy for asthma & BEAMON & {$[8]$} \\
Alexander technique for chronic asthma & DENNIS & {$[9]$} \\
Breathing exercises for asthma & HOLLOWAY & {$[10]$} \\
Family therapy for asthma in children & PANTON & {$[12]$}
\end{tabular}

\section{Homeopathy}

The founder of homeopathy was Samuel Hahnemann (1755-1843), a German doctor who reacted against the ineffective medicine of the time. The term homeopathy comes from the concept Homios (same and pathos), suffering. Homeopathy is based on the principle of diluting a substance that can cause a disease in a healthy person in undiluted form down to a solution that appears to cure the sufferer. The scale of dilution is, as a rule, extreme: often the active substance is diluted $>1$ million times, commonly up to $\times 10^{12}$ $(=1 / 1,000,000,000,000)$. The homeopath believes that dilution replaces material strength by a non-material force that has a positive effect on disease. As an example, patients with tuberculosis (TB) are treated with the expectorate of an individual with TB diluted $\sim 1$ billion times. Another less dramatic alternative is pollen allergy, which is treated with pollen extract diluted $>1$ million times.

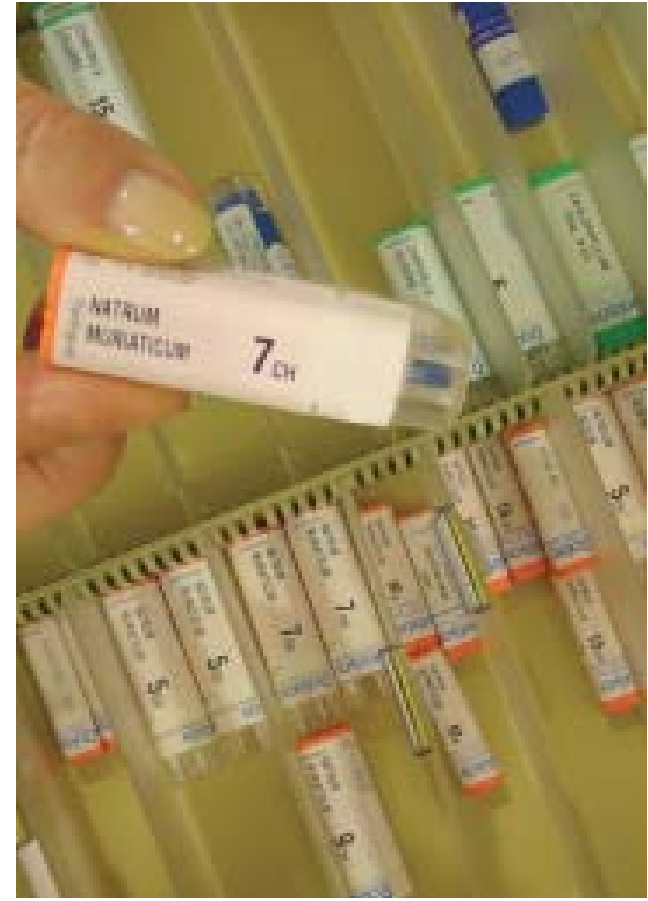

Six placebo-controlled studies analysing the effect of homeopathy on chronic asthma are included in the Cochrane review on this subject [2]. The observation period was at least 1 week. In all cases, homeopathy was used as a complement to orthodox medicine, e.g. inhaled steroid treatment. The six studies involved 556 patients in total. Most studies used lung function parameters, although a change in the need for medication was also analysed in some of the studies.

One common problem was that the treatment itself varied considerably between studies and from patient to patient. Consequently, it is not easy to give an opinion on whether one particular homeopathic medicine has more "effect" than another. The evaluative factor was, therefore, whether homeopathic treatment itself can have some positive effect. Another problem with these studies was that no attempt was made to discriminate precisely what was positive in the treatment, as the entire treatment situation itself (the treatmentenvironment) can be considered to have particular significance upon the outcome. One example is that patients who were given "active" therapy in one group were followed-up with frequent telephone contacts, which can, by itself, have a very positive effect.

Varying results relating to the effect of lung function, symptoms and the consumption of medicine were reported in the studies. The conclusion of the review was that no convincing effects of homeopathy on chronic asthma can be found. 
No systematic review has been published on homeopathy and allergic asthma. However, one large, placebo-controlled, multi-centred study has been carried out in the UK and is worth a mention [3]. The intention of this investigation was to study whether homeopathic treatment could have an effect on patients with allergic asthma who were sensitive to dust mites. A total of 242 patients were included and treated during a 16week period. No difference could be found in the development of lung function (forced expiratory volume in one second (FEV1)), peak expiratory flow variability, asthma symptoms or quality of life.

\section{Manual therapy}

Manual therapy is defined as the manual treatment of the chest or spine with the intention of achieving improved ventilation, reduced muscle spasm and mucous mobilisation. A number of physiotherapeutic techniques and manipulation of the spine (also known as chiropractic treatment) are included in this category.

In the Cochrane review [4], a total of 393 references were found in the literature, including 59 full-length articles. Among these were nine references to five clinical studies with acceptable designs. The latter five studies included a total of 290 patients. The studies were selected on the basis of the following necessary/essential criteria: they should be randomised; they should include asthmatic children or adults; they should investigate the effect of one or two manual therapies comparing one with the other; and they should include relevant clinical parameters for the evaluation of effect.

Out of the five studies, two were "so-called" chiropractic manipulation. Both were of good scientific quality and neither of them showed any effect of manipulation compared with "placebo treatment". A smaller study compared massage treatment with a control group who were offered general relaxation treatment. Thirty-two children were treated with massage for 20 minutes. A small but significant improvement was found in one of the lung function parameters (forced midexpiratory flow) in the treatment group. It is unclear, however, in this case if there was correction for so-called multiple comparisons. Thus, this may possibly be due to a statistical error. In the last study, conventional physiotherapy was compared with a placebo treatment and reflexology in an open non-controlled design. No possible positive effect could be documented in any of these studies.

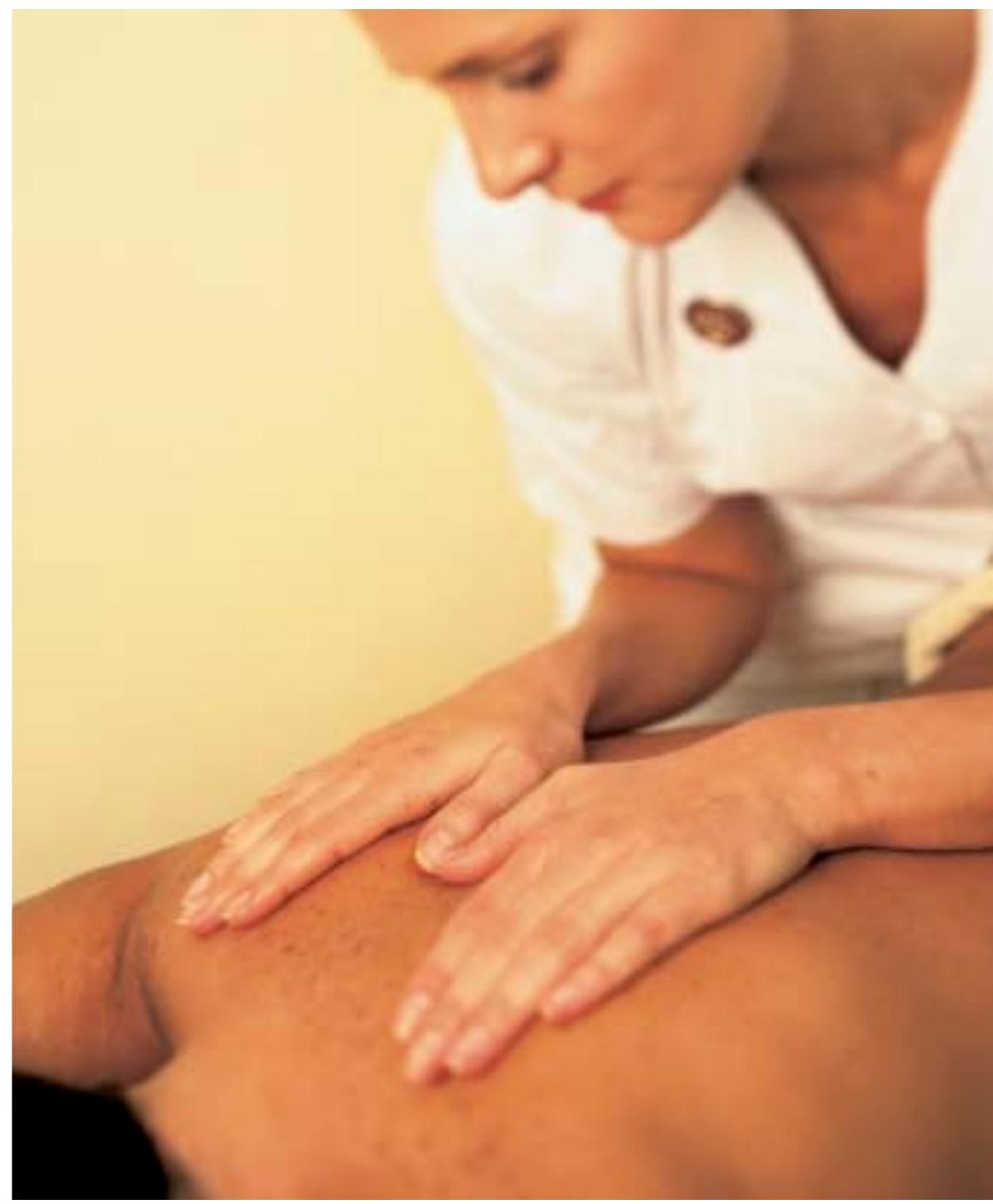

\section{Acupuncture treatment}

Acupuncture treatment has its roots in traditional Chinese medicine. Acupuncture theoretically holds that the body consists of a network of meridians through which life energy, qi, is flowing. When one is in good health qi flows harmoniously. During illness, blockages or irregularities occur in the flow. By stimulating these meridians at different acupuncture points, one can restore the balance and thus free the patient from illness.

The criteria for including studies for analysis in this review [5] were as follows. Randomised studies in which puncture with needles or other forms of stimulation of acupuncture points were used, and they should, in addition, include a control arm, not necessarily a placebo. The control arm could also be the so-called "traditional treatment". Furthermore, assessable parameters and a treatment duration of at least 1 week were required. In 


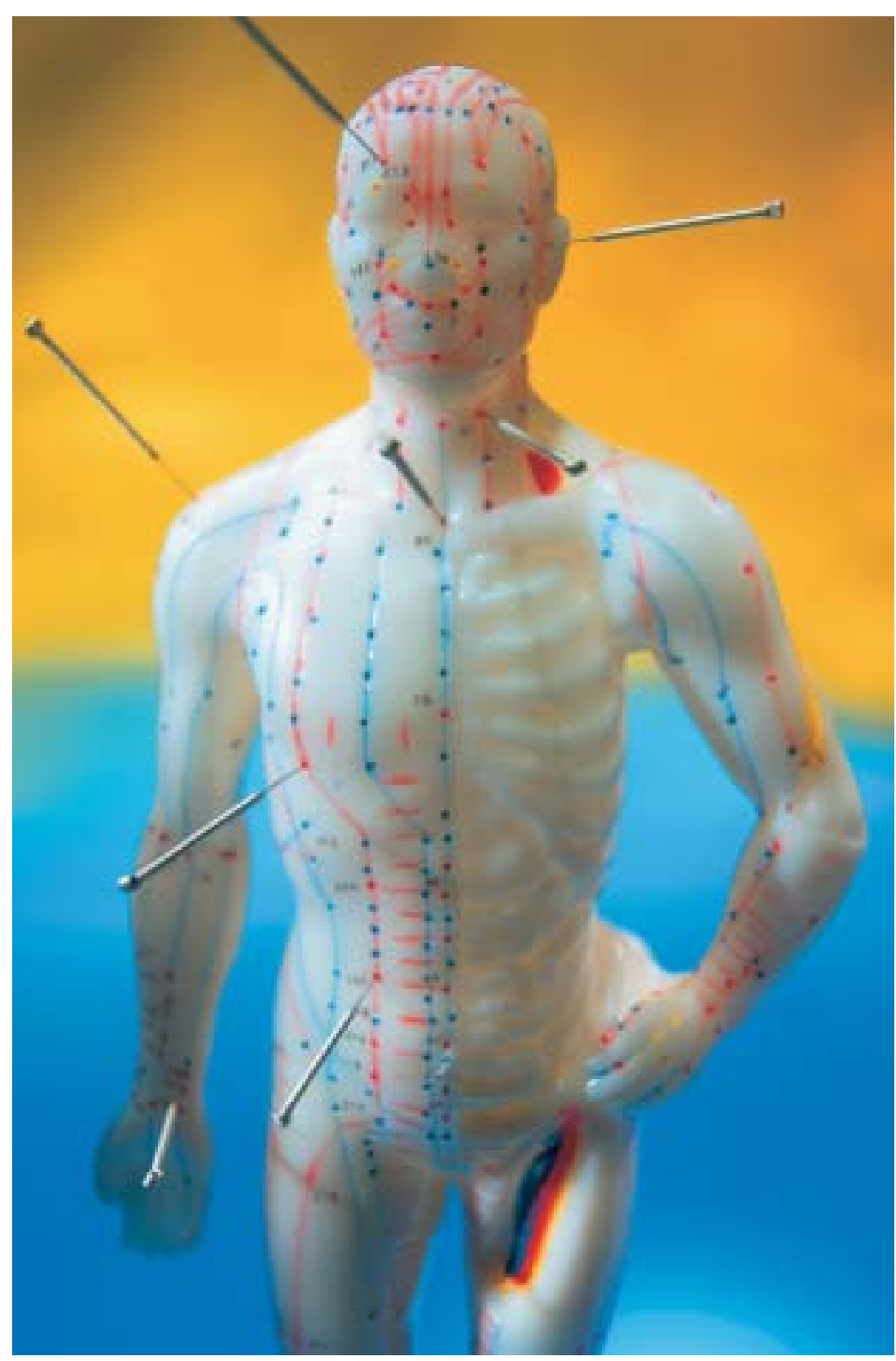

total, 11 studies were found, including 324 patients who met the acceptability quality criteria. In two of the studies, more formalised acupuncture was used, with stimulation of predetermined points, which were compared with placebo points. None of the studies could demonstrate any clinically relevant effect compared with the control arm. In two of the studies, lung function data were pooled and showed a tendency towards a slight increase in $\mathrm{FEV}_{1}$; however, this was not significant.

In conclusion, in the scientific studies carried out, there was no proof of a clinically relevant or significant effect of treatment by acupuncture on chronic asthma.

\section{Ionisation of air}

This method of treatment is based on a correct scientific observation, where it was found that the ion content of inhaled air can affect lung function. On this basis, attempts were made to manufacture so-called air ionisers, which are supposed to "de-ionise" the air and thereby achieve a better respiratory atmosphere. The idea is that one may, in this way, reduce the risk of developing allergies or asthma.

In the present review [6], the effect of treatment with an ioniser apparatus was compared with so-called "dummy ionisers". In the dummy ionisers, the motors of the ionisers were, in principle, running, but without having any de-ionising effect. The selection criteria were consequently placebo-controlled, randomised studies in the treatment of children or adults with chronic asthma.

A total of six studies were found in the literature that met the inclusion criteria. The studies involved a total of 106 patients. None of the studies showed any positive effect in the form of a change in the need for medication during an attack or symptom control. One study showed a tendency for ionised air to bring on a more serious attack of FEV1 after physical exertion, but the results were never statistically significant [7].

The generic assessment is, consequently, that the de-ionising of air using so-called ionising apparatus has no effect on chronic asthma in children or adults.

\section{Speleotherapy}

This form of treatment has its origins in East and Central Europe where, for many years, both children and adults with lung diseases and rheumatic disorders etc. have been treated by daily sessions in caves. The idea is that the special air inside the caves, which has high humidity and a high mineral content, has a beneficial effect on several different illnesses. Patients dressed in special equipment with warm clothes and safety helmets rest in beds inside the caves. There is also a company that specialises in this kind of treatment and manufactures special salt chambers where one can sit in a room filled with salt and minerals on the walls.

Studies dealing with speleotherapy were included for analysis in this review [8] provided that there was some comparison of effect between speleotherapy and some other intervention or, alternatively, absence of intervention in patients with chronic asthma. Three studies met minimal criteria for analysis, including a total of 
124 asthmatic children. Only one of the studies was considered to have sufficient methodological quality; two of the studies reported that speleotherapy had a short-lived, temporary effect on lung function, but, for the rest, there were no signs of a positive effect of the treatment.

The conclusion is that there is no available evidence to reinforce the hypothesis that speleotherapy can have any positive effect in the treatment of chronic asthma. Randomised studies with follow-up over a longer period are required if any conclusions are to be drawn.

\section{The Alexander technique}

The Alexander technique is a method developed by the actor Fredril Mattias Alexander (1869-1955). The method is based on the idea of increasing one's knowledge of the body and movement patterns so that one can avoid having a bad back, stiff neck and headache. Advocates of the technique believe that asthma symptoms and control of asthma can be improved by increasing relaxation of the body and by an improved knowledge of the body and bodily control.

Criteria for inclusion and analysis in the review [9] were randomised, controlled studies where the Alexander technique was used with the aim of ameliorating symptoms or control of disease in patients with chronic stable asthma. Either another intervention or no intervention was used as comparison.

Unfortunately, no studies found met the criteria to produce reliable analyses. The conclusion of the review was that scientific studies indicating whether or not the Alexander technique has any effect are lacking.

\section{Breathing exercises}

A tradition exists that breathing exercises can ameliorate chronic asthma, both in eastern and western medicine, and there is a wealth of socalled anecdotal evidence.

Some 42 studies were evaluated for inclusion in this systematic review [10]. A total of 35 of these studies were excluded and, therefore, a total of seven studies were finally included. Most of these studies were on a small scale. Two studies showed significant reduction in the use of bronchodilating medication for improving symptoms ("rescue medication") and three studies showed a reduction in the number of exacerbations. Two single studies showed improved quality of life. In general, a positive effect of breathing exercises was found. Breathing exercises can be classified as complementary therapy, but not as alternative treatment.

\section{Family therapy}

There is little doubt that psychosocial and emotional factors are important in the treatment of asthma. Treatment programmes that include behavioural and psychological interventions have been developed to improve disrupted family relations in families with children who have serious asthma. STRUNK etal. [11] have shown that family relationships and social mastering are important for the long-term prognosis for children with serious asthma in an investigation of children who died of asthma.

Two studies with a total of 55 children were included in this Cochrane review [12]. However, the results from these two studies could not be combined in a rational way. In one study, the gas volume, peak expiratory flow rate and breathing difficulty during the day were improved in the treated children when compared to controls. In the other study, an improvement was observed in total clinical evaluation and number of days with reduced functioning. The authors concluded that family therapy can be a valuable complement to medical treatment.

\section{Acupuncture and smoking cessation}

Smoking, either active or passive, has a deleterious effect on asthma patients and also on lung function in the very young [13]. Smoking cessation is, therefore, very important for this group of

\section{Educational questions}

1. What is the main explanation for the difficulty in documenting the effects of CAM in patients with asthma and/or allergy?

2. What is the basic theory behind homeopathy?

3. What does the Alexander technique stand for?

4. How could one design better studies to determine whether CAM is effective or not? 
patients. Acupuncture has been claimed to help patients stop smoking. This has also been the subject of a systematic Cochrane review [14].

Some 22 studies were identified, although it was not possible to prove any effect of acupuncture in smoking cessation when compared with simulated acupuncture ("sham acupuncture"). The odds ratio (95\% confidence intervals) for positive early result was $1.22(0.99-1.49)$ for 6 months' follow-up and 1.50 (0.99-2.27) for 12 months' follow-up. No significant differences could be found and no significant effect could be seen using various acupuncture treatment programmes alone.

\section{Conclusions}

Systematic analysis of scientific results in accordance with Cochrane's methods is, in the context of orthodox medicine, a well-documented method for establishing whether a treatment has an effect or not. In these systematic reviews carried out on a number of alternative medical methods, there has been no proven effect of any such treatment, although this does not mean that it has been proven that there is no effect. What can be said is that the supporting evidence existing today is not sufficient to allow one to conclude whether the methods are effective or not.

One problem with alternative medicine is the difficulty to reach a consensus concerning the very choice of method. It is often hard to sift out exactly what it is in the treatment itself that constitutes the effective component. In homeopathy, and even in acupuncture treatment, one would like to give so-called personalised treatment in which the treatment strategy is individually chosen after an initial overall assessment of the patient's condition. Using this perspective, asthma does not become one or several illnesses, but only a component in a holistic picture of the single individual. Each individual is unique. Consequently, one does not study the effect of a particular treatment of the condition known as asthma, but rather the effect of a therapeutic principle on different patients who have the diagnosis of asthma in common.

Another problem with alternative medicine is the difficulty of distinguishing between the effect of the treatment perse and the effect of the treatment situation and the therapist her/himself. Most often, a total concept is offered where the patient trusts the therapist conducting the treatment. The placebo effect is used more or less consciously.

Some of the studies presented have been of extraordinarily high scientific quality with exemplary randomisation and placebo controls. None of these studies have been able to show any positive effect. They have, however, demonstrated that it is possible to carry out correct scientific studies and, thus, it is strongly recommended that, even in alternative medicine, one should strive to document the effect of the treatment principles. 


\section{Suggested answers}

1. The therapeutic environment is unique, and it is often impossible to differentiate between the effect of the treatment itself and the effects of the therapist and the environment associated with him/her.

2. "Cure disease with disease". By extreme dilution of a substance that can cause disease in an undiluted form, homeopathy believes that you may create a solution that can cure the disease.

3. Alexander is named after the developer, Fredrik Mattias Alexander (1869-1955). The aim of the technique was to achieve better disease control by raising awareness of the body and, therefore, avoiding muscle stiffness and improving bodily control. In some way, the technique has much in common with yoga.

4. It is difficult, but not impossible, to design a controlled clinical trial to investigate the effects of CAM. However, the evaluation variables need to be defined and, as stated above, it is important to distinguish between the effect of the treatment itself and the effect of the treatment environment and the handing of the patient.

\section{References}

1. Harbour R, Miller J. A new system for grading recommendations in evidence based guidelines. BMJ 2001; 323: 334-336.

2. McCarney RW, Lind K, Lasserson TJ. Homeopathy for chronic asthma. Cochrane Database Syst Rev 2004; 4: CD000353.

3. Lewith GT, Watkins $A D$, Hyland ME, et al. Use of ultramolecular potencies of allergen to treat asthmatic people allergic to house dust mite: double blind randomised controlled clinical trial. BMJ 2002; 324: 520.

4. Hondras MA, Linde K, Jones AP. Manual therapy for asthma. Cochrane Database Syst Rev 2002; 4: CD001002.

5. McCarney RW, Brinkhaus B, Lasserson TJ, Linde K. Acupuncture for chronic asthma. Cochrane Database Syst Rev 2004; 1: CD000008.

6. Blackhall K, Appleton S, Cates CJ. Ionisers for chronic asthma. Cochrane Database Syst Rev 2003; 3: CD002986.

7. Wagner CJ, Danziger RE, Nelson HS. Relation between positive small air ions, weather fronts and pulmonary function in patients with bronchial asthma. Ann Allergy 1983; 51: 430-435.

8. Beamon S, Falkenbach A, Fainburg G, Linde K. Speleotherapy for asthma. Cochrane Database Syst Rev 2001; 2: CD001741.

9. Dennis J. Alexander technique for chronic asthma. Cochrane Database Syst Rev 2000; 2: CD000995.

10. Holloway E, Ram FS. Breathing exercises for asthma. Cochrane Database Syst Rev 2004; 1: CD001277.

11. Strunk RC, Mrazek DA, Fuhrmann GS, LaBrecque JF. Physiologic and psychological characteristics associated with deaths due to asthma in childhood. A case-controlled study. JAMA 1985; 254: 1193-1198.

12. Panton J, Barley EA. Family therapy for asthma in children. Cochrane Database Syst Rev 2000; 2: CD000089.

13. Lødrup Carlsen KC, Jaakkola JJ, Nafstad P, Carlsen KH. In utero exposure to cigarette smoking influences lung function at birth. Eur Respir J 1997; 10: 1774-1779.

14. White AR, Rampes H, Ernst E. Acupuncture for smoking cessation. Cochrane Database Syst Rev 2002; 2: CD000009. 